\title{
A Super-resolution Algorithm for Enhancement of FLASH LIDAR Data: Flight Test Results
}

\author{
Alexander Bulyshev*a, Farzin Amzajerdian ${ }^{\mathrm{b}}$, Eric Roback ${ }^{\mathrm{b}}$, and Robert Reisse ${ }^{\mathrm{b}}$ \\ a Analytical Mechanical Associates, Inc, Hampton, VA23666; ' $\mathrm{NASA}$ Langley Research Center, \\ Hampton, VA 23681.
}

\begin{abstract}
This paper describes the results of a 3D super-resolution algorithm applied to the range data obtained from a recent Flash Lidar helicopter flight test. The flight test was conducted by the NASA's Autonomous Landing and Hazard Avoidance Technology (ALHAT) project over a simulated lunar terrain facility at NASA Kennedy Space Center. ALHAT is developing the technology for safe autonomous landing on the surface of celestial bodies: Moon, Mars, asteroids. One of the test objectives was to verify the ability of 3D super-resolution technique to generate high resolution digital elevation models (DEMs) and to determine time resolved relative positions and orientations of the vehicle. 3D super-resolution algorithm was developed earlier and tested in computational modeling, and laboratory experiments, and in a few dynamic experiments using a moving truck. Prior to the helicopter flight test campaign, a 100mX100m hazard field was constructed having most of the relevant extraterrestrial hazard: slopes, rocks, and craters with different sizes. Data were collected during the flight and then processed by the super-resolution code. The detailed DEM of the hazard field was constructed using independent measurement to be used for comparison. ALHAT navigation system data were used to verify abilities of super-resolution method to provide accurate relative navigation information. Namely, the 6 degree of freedom state vector of the instrument as a function of time was restored from super-resolution data. The results of comparisons show that the super-resolution method can construct high quality DEMs and allows for identifying hazards like rocks and craters within the accordance of ALHAT requirements.
\end{abstract}

\section{INTRODUCTION}

The term super-resolution (SR) applied to image reconstruction generally refers to the group of signal processing algorithms that improve the spatial resolution of an image to better than the size of the pixel of the original image. This technique is implemented by taking advantage of sub-pixel shifts between multiple, low-resolution images of the same scene. SR is a wellestablished technique for enhancing two-dimensional (2-D) images and over the years, a large number of algorithms have been developed for processing intensity images produced by different types of imaging systems [1-5]. The emergence of Flash Lidar technology that is capable of generating three-dimensional (3-D) images at video rates, with a relatively large number of pixels has created a need to extend 2-D SR techniques to 3-D images [7-13]. The key technological breakthrough was the development of highly sensitive Avalanche Photodetector (APD) arrays and associated Readout Integrated Circuit (ROIC) having a relatively large number of pixels. Unlike earlier imaging Lidar systems that generate 3-D images of a scene by scanning the laser beam across the targeted scene and measuring the time of arrival for each returned laser pulse, the Flash Lidar records a full 3-D image using a single laser pulse for the entire focal plane array. Therefore, the Flash Lidar permits much higher frame rates without any blurring or inaccuracies due to the platform motion. 
The ability of Flash Lidar to generate 3-D images at relatively high frame rates and with a relatively large number of pixels from a moving platform has created a new set of applications. For example, the NASA's Autonomous Landing and Hazard Avoidance Technologies (ALHAT) project is developing a Flash Lidar sensor that detects hazardous terrain features such as craters, rocks, and slopes during the terminal descent phase of a spacecraft when landing on planetary bodies [6]. Through theoretical and simulation analysis, the ALHAT team has determined that a single frame of Flash Lidar data may not be sufficient to build a landing site digital elevation map (DEM) with acceptable spatial resolution, precision and area size that will meet current system requirements. Similarly, building a mosaic of multiple frames with acceptable spatial resolution and size may not be possible within the short time window available to detect hazards prior to touchdown. To overcome this limitation, we developed a new SR method to enhance the resolution of individual Flash Lidar 3D image frames and generate a sufficiently large DEM [14].

Over approach is can be divided into three major blocks: 3D modification of back projection method [11], 6D version of the Lucas-Kanade registration algorithm [15], and the modified inverse filtering algorithm $[13,16]$. This paper first describes the static test data gathered at the NASA Langley Research Center Lidar Test Range facility. The dynamic test data obtained during a helicopter flight test of the same Flash Lidar is then reported. The image resolution enhancement and noise reduction and the determination of the platform position and angular orientation resulting from the processing of the Flash Lidar range image data by the SR algorithm are explained.

\section{Ground Test}

A series of tests were conducted using the ALHAT Flash Lidar at the NASA Langley Research Center (LaRC) Long Range Test Facility. The general view of the measuring set up is shown in Figure 1. ALHAT Flash Lidar system and performance specifications are summarized in Table 1.

Table 1. Specifications of the ALHAT Flash Lidar.

\begin{tabular}{|l|l|}
\hline Parameter & Value \\
\hline Array Size & $128 X 128$ \\
\hline Operational Wavelength & 1.06 micron \\
\hline Laser Pulse energy & $50 \mathrm{~mJ}$ \\
\hline Laser Pulsewidth & $8 \mathrm{nsec}$ \\
\hline Receiver Field of View & 1 deg. and $5 \mathrm{deg}$ \\
\hline Receiver Aperture Diameter & $100 \mathrm{~mm}$ (75 mm for 5 deg lens) \\
\hline Frame Rate & $20 \mathrm{~Hz}$ \\
\hline \multirow{2}{*}{$\begin{array}{l}\text { Max operational range (natural } \\
\text { terrain target) }\end{array}$} & $1500 \mathrm{~m} @ 90^{\circ}$ look angle \\
\cline { 2 - 2 } & $950 \mathrm{~m} @ 30^{\circ}$ look angle \\
\hline Range Precision & $8 \mathrm{~cm}$ \\
\hline
\end{tabular}



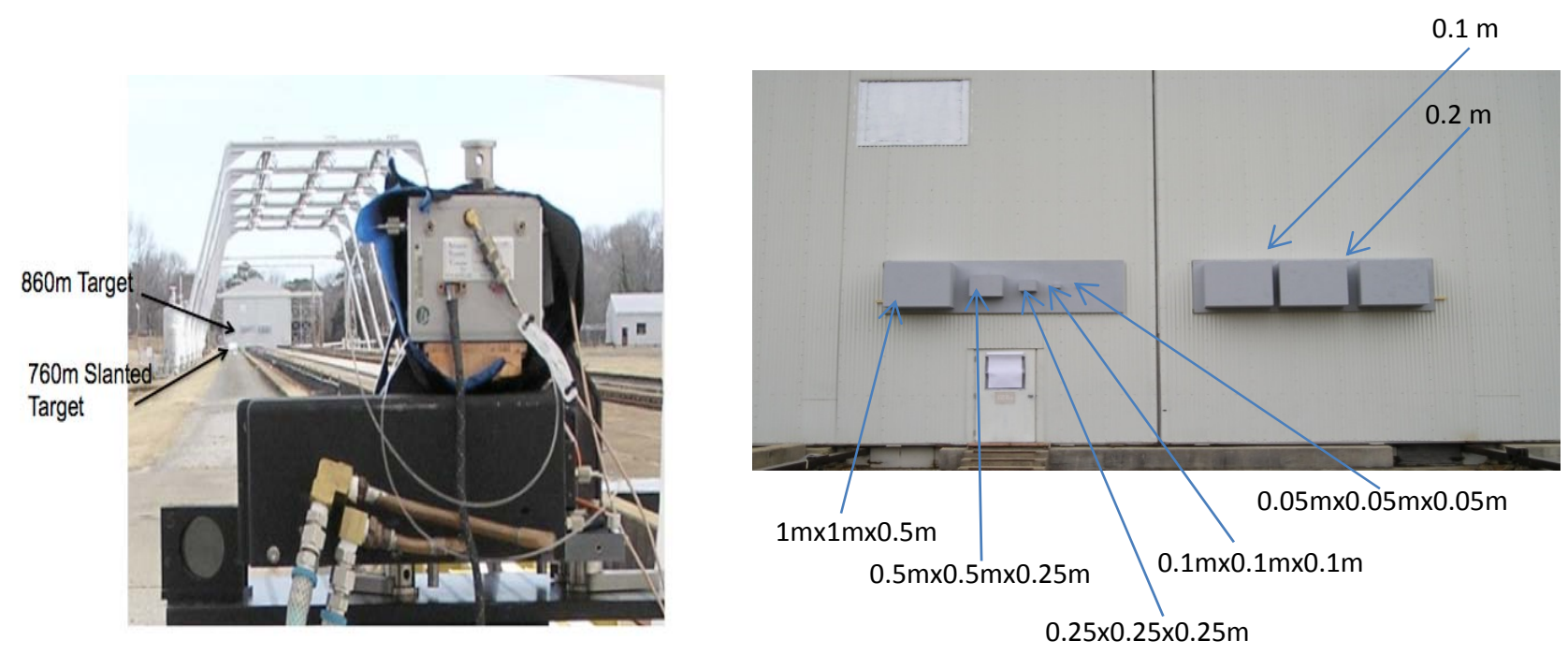

Figure 1. a) general view of the experimental setup; b) lidar target .

The lidar target was actually a flat wall about $15 \mathrm{mX} 20 \mathrm{~m}$ in dimensions with a number of calibrated rectangular boxes attached to it. The sizes of boxes and distances between them are indicated in Figure1 (b). The major purposes of the experiments were to test the detectability limits of the SR algorithm using the small boxes on the left side of the scene, and to quantify the spatial resolution enhancement of the algorithm using the gaps between the boxes on the right side of the scene. The Lidar FOV was 1 degree for this experiment. The results of restoration are presented in Figure 2.

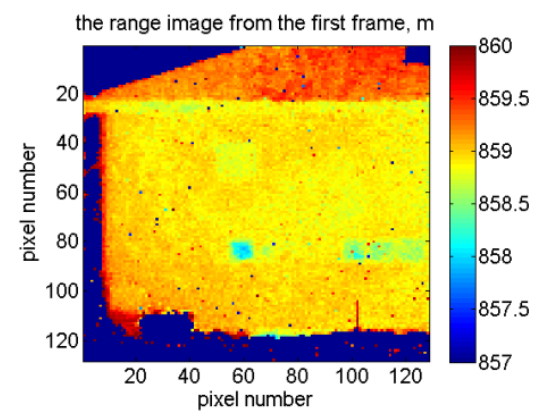

a)

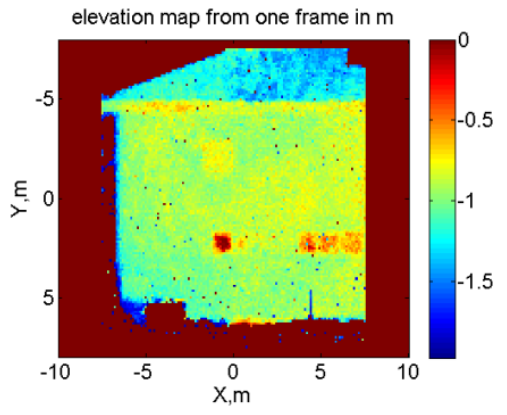

b)

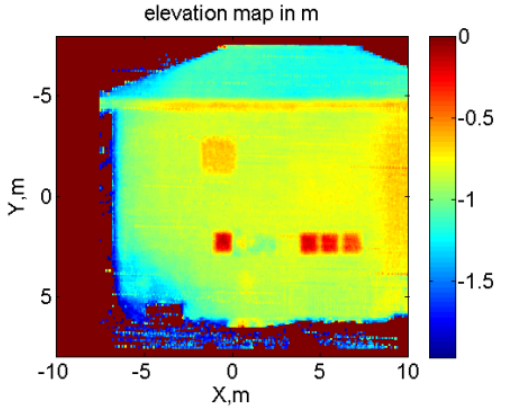

c)

Figure 2. a) Range image; b) DEM obtained using 1 frame; c) DEM obtained using 20 frames.

The effective lidar pixel footprint on the target is $12 \mathrm{~cm}$ from $860 \mathrm{~m}$ distance. The smallest box that can be identified with a high level of certainty is $0.25 \mathrm{~m}$ in dimension. The gap between the two 10-cm boxes is also very recognizable. Neither of these features can be identified in the single frame image due to low resolution and presence of range noise (about $8 \mathrm{~cm}$ ). The significant improvement resulting from application of the SR algorithm is clear when comparing the single frame image with the SR processed image of Figure 2.

\section{Helicopter Flight Test}


A helicopter flight test was conducted in December 2012 under the ALHAT project over a simulated lunar terrain site consisting of realistic hazard features constructed near the Space Shuttle Landing Facility at Kennedy Space Center (KSC). One of the objectives of the test was to verify the ability of the SR algorithm to build a high resolution map and to demonstrate its relative navigation capabilities in a dynamic flight environment. The general view of the field is shown in Figure 3.

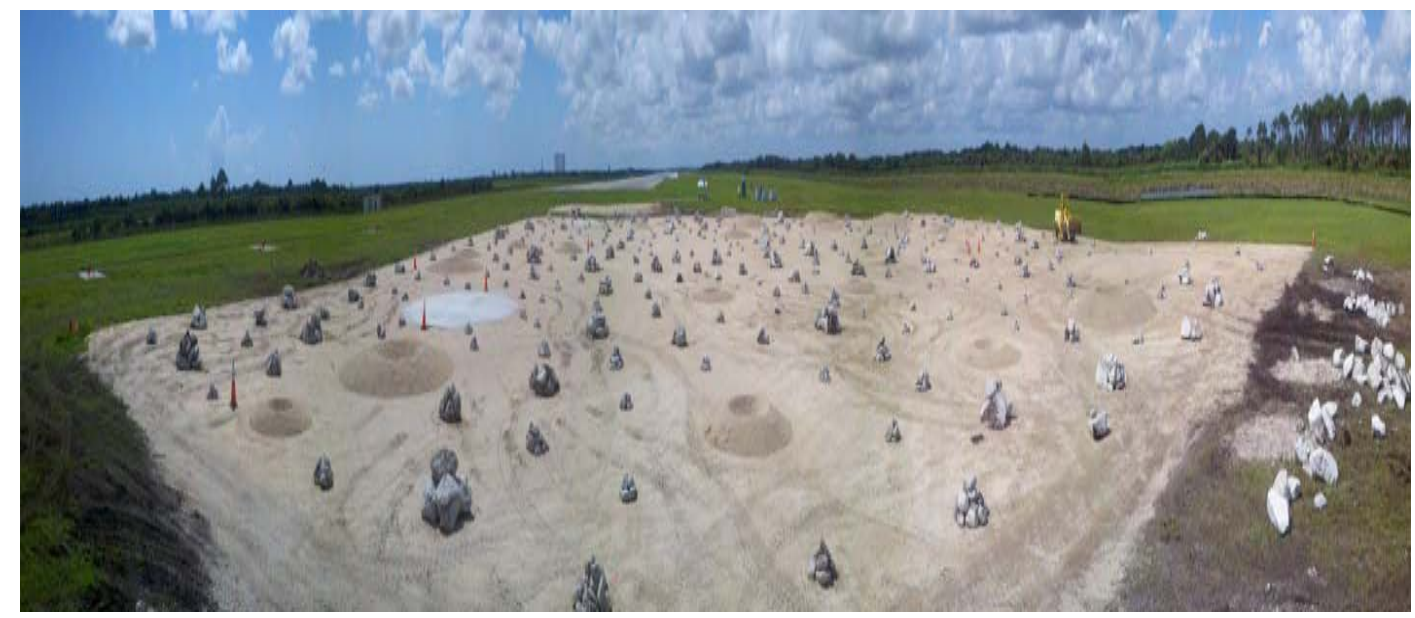

Figure 3. ALHAT Hazard Field at NASA KSC

The hazard field was surveyed and a precise DEM with $10 \mathrm{~cm}$ resolution was prepared. This DEM was used as the truth DEM for analyzing the Flash Lidar data with and without SR processing. The test parameters were: Flash Lidar FOV 5 degrees, slant range from $1 \mathrm{~km}$ to $50 \mathrm{~m}$, and look angle close to 30 degree. Figure 4 illustrates the results obtained from 400 slant range. In addition to noise suppression and improving spatial resolution, the current example demonstrates another super-resolution attribute - ability to add missing data from different frames.

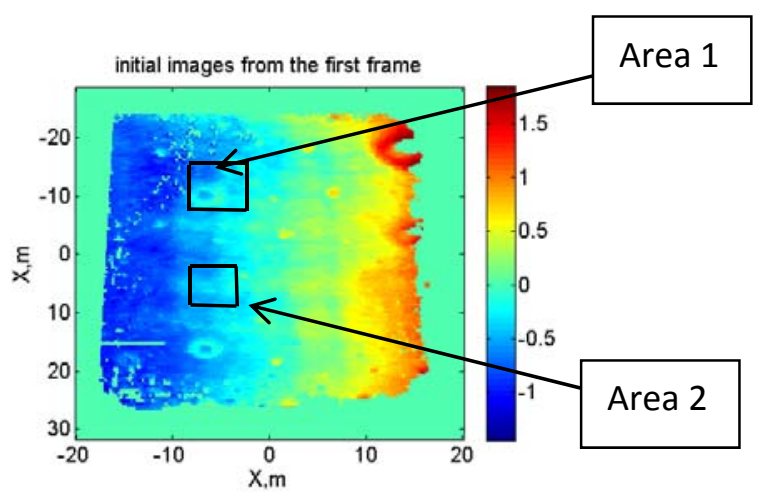

a)

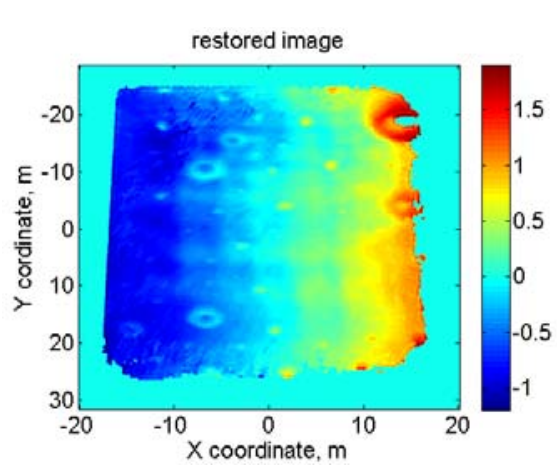

b)

Figure 4. Results of DEM restoration: a) DEM obtained using 1 frame; b) SR DEM obtained from 20 frames. Zoomed Area1 and Area2 are presented in Figures 5-6. 
DEM obtained from a single frame (Fig.4 a) contains many blank spots. These "bad pixels" are the reflection of non-responding detectors. Most of these spots are compensated by data added from other frames.

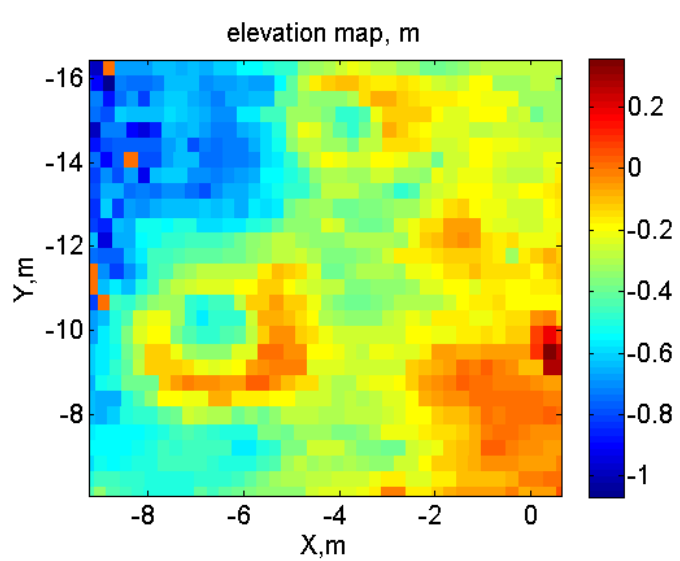

a)

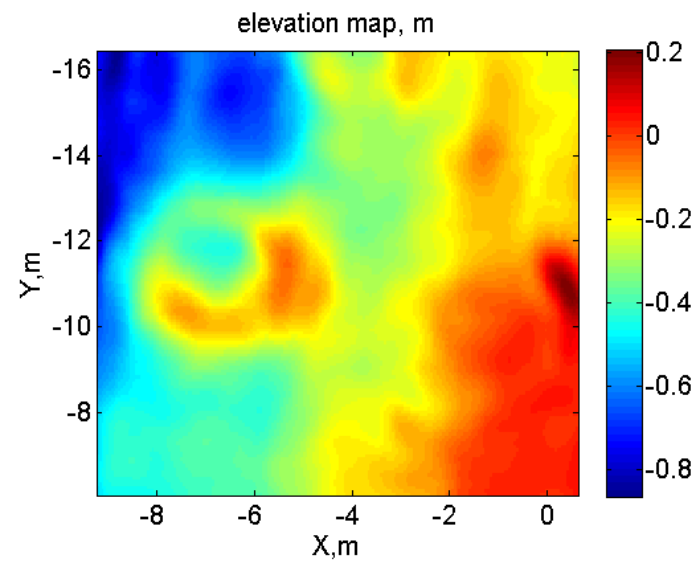

b)

Figure 5. Extended Area 1; a) DEM obtained from 1 frame; b) SR DEM obtained from 20 frames.

Figure 5 demonstrates how small scale structures become identifiable and the image noise is reduced. The extended area 2 as shown in Figure 6 demonstrates that the hazards (stones) with diameter $0.4 \mathrm{~m}$ and height $0.2 \mathrm{~m}$ can be identified.

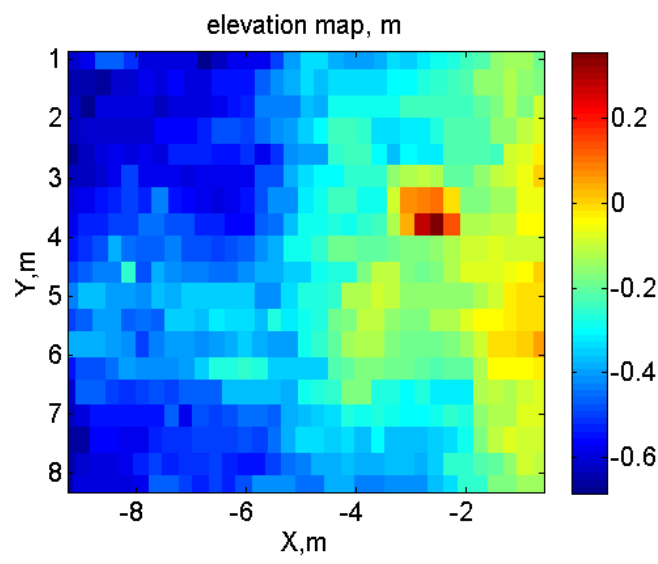

a)

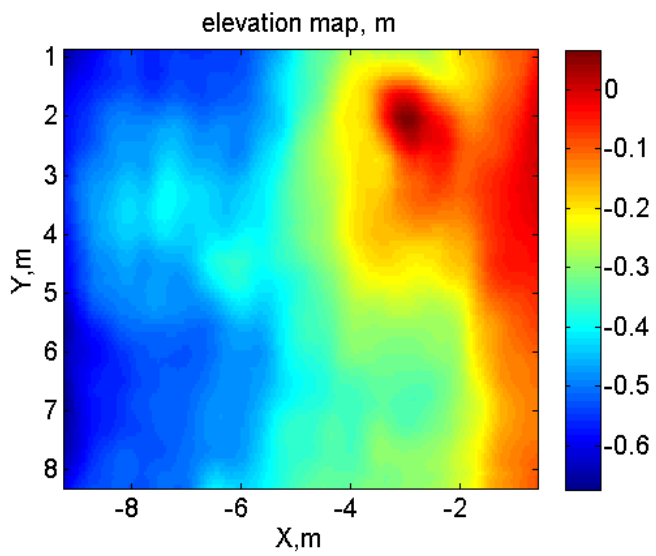

b)

Figure 6. Extended Area 2; a) DEM obtained from 1 frame; b) SR DEM obtained from 20 frames.

Comparing the true DEM with the restored SR DEM, as illustrated in Figure 7, reveals the merits of this image enhancement technique. 


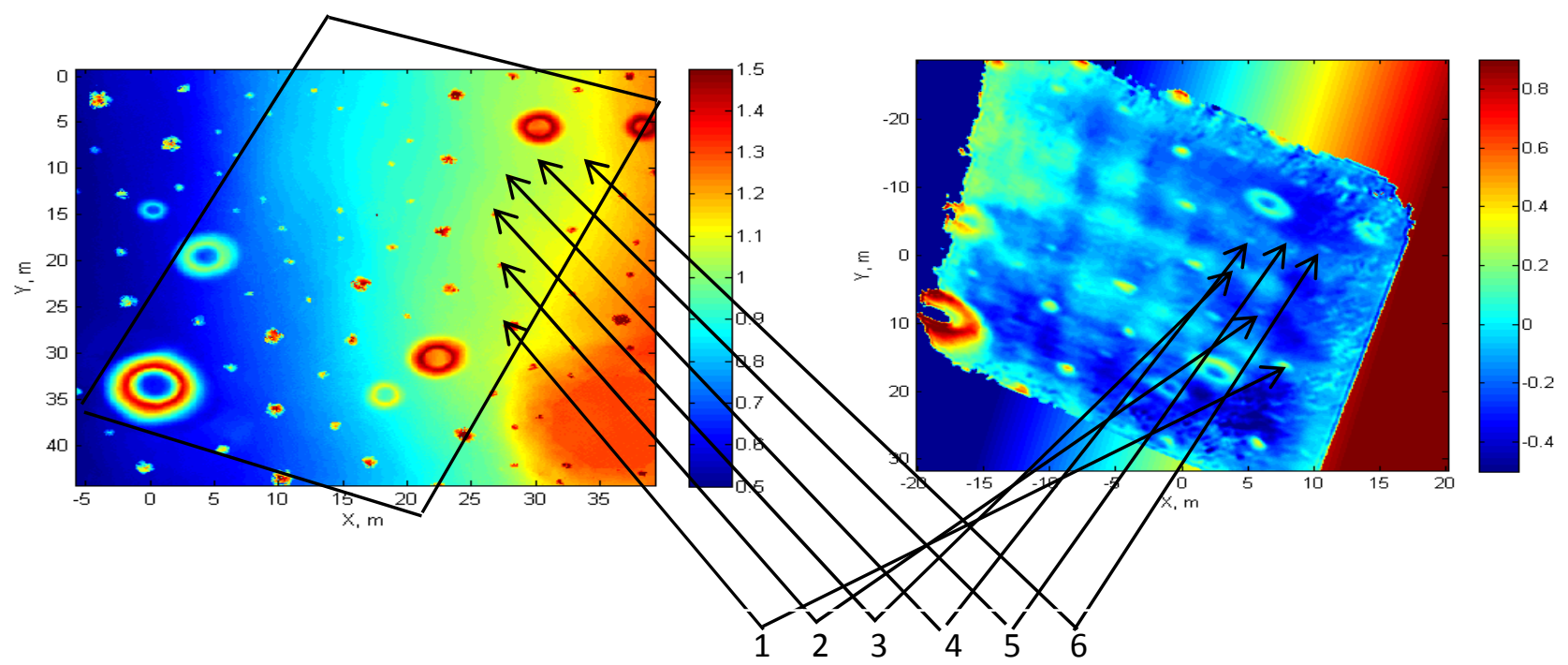

Figure 7. Comparison of the truth DEM and restored DEM

The black rectangular on the true DEM shows position and orientation of the restored DEM. All large scale features like big craters and rocks are very visible and located in the same positions as they are located in the true DEM. Six small features (rocks) are compared in details individually. Arrows in Figure 7 point to a set 6 distinguishable rocks in the truth DEM and their corresponding image in Flash Lidar data. The table 2 lists the measured diameters and heights of these 6 rocks from the truth map with the measurements obtained from SR processed Flash Lidar data. Diameter is counting as the average size in two horizontal orthogonal directions close to a rock base, and height is the highest point. Five of the six rocks can be identified with high certainty. One which cannot be identified is smaller than $30 \mathrm{~cm}$.

Table 2. Comparison of Flash Lidar SR-processed data with Truth DEM.

\begin{tabular}{|c|cc|cc|}
\hline rock number & \multicolumn{2}{|c|}{ Diameter, m } & \multicolumn{2}{|c|}{$\begin{array}{c}\text { Height, m } \\
\text { Restored }\end{array}$} \\
\hline 1 & 1.3 & 1.3 & 0.6 & 0.5 \\
\hline 2 & 0.8 & 1.2 & 0.3 & 0.12 \\
\hline 3 & 0.7 & 1.0 & 0.4 & 0.21 \\
\hline 4 & 0.6 & 1.0 & 0.2 & 0.3 \\
\hline 5 & 0.6 & - & 0.25 & - \\
\hline 6 & 0.7 & 0.6 & 0.27 & 0.2 \\
\hline
\end{tabular}

\section{Conclusions}


A novel truly 3D super-resolution algorithm has been conceived, implemented, and tested. A series of computer modeling experiments, laboratory experiments, and field tests were conducted demonstrating the algorithm's ability to build high resolution 3D images based on processing multi frames data sets of the same object. The method includes 6D image registration algorithm, which itself provides high quality relative navigation data. The SR algorithm has been applied to the 3-D image data using a Flash Lidar instrument with a 128X128 pixels detector array, developed by NASA LaRC for ALHAT project. The SR algorithm shows 4X digital magnification (using 20 frames), relative navigation accuracy of about $30 \mathrm{~cm}$, significant noise suppression feature by a factor of 4 to 5 times, and ability to compensate for the missing pixel data.

\section{References}

1. S. Park, M. K. Park, and M. Kang, “Superresolution image reconstruction: A technical review,” IEEE Signal Processing Magazine 20(3), 21-36, (2003).

2. S. Farsiu, M. D. Robinson, M. Eland, and P. Malinfar, "Fast and robust multiframe super resolution,” IEEE Transactions on Image Processing 13(10), 1327-1344, (2004).

3. S. Young and R. Driggers, "Superresolution image reconstruction from a sequence of aliased imagery,” Applied Optics 45(21), 5073-5085, (2006).

4. G. Clement, J. Huttunen, and K. Hynynen, "Superresolution ultrasound imaging using backprojection reconstruction,” Journal of the Acoustical Society of America 118(6), 3953-3960, (2005).

5. S. Chaundhuri, Super-resolution Imaging, Springer, 2001.

6. C. Epp, E. Robinson, and T. Brady, “Autonomous landing and hazard avoidance technology,” in Proceedings of IEEE Aerospace Conference, 659-665,(2008).

7. Q. Yang, R. Yang, J. Davis, and D. Nister, "Spatial-depth super resolution for range images," in Proceedings of IEEE Conference on Computer Vision and Pattern Recognition, 1-8, (2007). 8. G. Rosenbush, T. Hong, and R. Eastman, "Super-resolution enhancement of flash LADAR range data," in Proceedings of SPIE, Unmanned/Unattended Sensors and Sensors Networks IV, 6736, 673614-1 - 673614-10, (2007).

9. S. Hu, S. Young, T. Hong, J. Reynolds, K. Krapels, B. Miller, J. Thomas, and O. Nguyen, "Super-Resolution for flash LADAR data," in Proceedings of SPIE, Infrared Imaging Systems: Design, Analysis, Modeling, and Testing XX, 7300, 73000-B1 - 73000B-12, (2009).

10. S. Hu, S. S. Young, T. Hong, J. Reynolds, K. Krapels, B. Miller, J. Thomas, and O. Nguyen, "Super resolution for flash LADAR imagery,” Applied Optics 49(5),772-780, 2010.

11. F. Qin, X. He, W. Chen, X. Yang, and W. Wu, "Video superresolution reconstruction based on subpixel registration and iterative back projection,” Journal of Electronic Imaging 18(1), 0130007, (2009).

12. J. Woods, E. Armstrong, W. Armbruster, and R. Richmond, "The application of iterative closest point (ICP) registration to improve 3D terrain mapping estimates using the flash 3D ladar system,” in Proceedings of SPIE, Laser Radar Technology and Applications XV, Orlando, Florida, 7684, 76840N,(2010).

13. E. Armstrong, R. Richmond, "The application inverse filter to 3D Microscanning of LADAR Imagery,” in Proceedings of IEEE Aerospace Conference, Big Sky, MO,(2006). 
14. Alexander Bulyshev, Michael Vanek, Farzin Amzajerdian, Diego Pierrottet, Glenn Hines, and Robert Reisse, "A super-resolution algorithm for enhancement of FLASH LIDAR data," Proc. SPIE 7873, 78730F, Feb (2011).

15. B. Lucas, T. Kanade, "An Iterative Image Registration Technique with Application to Stereo Vision,” in Proceedings of Imaging Understanding Workshop, 121-130, (1981). 16. M. Bertero, P. Boccacci, Introduction to Inverse Problems in Imaging. Bristol: IOP Publishing, (1998). 